\title{
Predicting Overall Survival Using Preoperative Nutritional and Inflammation Status for Colorectal Cancer
}

\author{
TAMURO HAYAMA ${ }^{1}$, TSUYOSHI OZAWA ${ }^{1}$, MITSUO TSUKAMOTO ${ }^{1}$, \\ YOSHIHISA FUKUSHIMA ${ }^{1}$, RYU SHIMADA ${ }^{1}$, KEIJIRO NOZAWA ${ }^{1}$, KEIJI MATSUDA ${ }^{1}$, \\ SHOICHI FUJII ${ }^{2}$, TAKEO FUKAGAWA ${ }^{1}$ and YOJIRO HASHIGUCHI ${ }^{1}$ \\ ${ }^{1}$ Department of Surgery, Teikyo University School of Medicine, Tokyo, Japan; \\ ${ }^{2}$ Department of Surgery, Yokohama General Hospital, Yokohama, Japan
}

\begin{abstract}
Aim: It has been shown that nutritional status and inflammation correlate with survival in patients with various cancer types. In this study, we evaluated several kinds of nutritional and inflammation parameters in preoperative blood samples and constructed new risk model predicting survival in patients with colorectal cancer. Patients and Methods: We retrospectively examined 286 patients with stage I-III colorectal cancer who had undergone curative resection at Teikyo University Hospital. The association between overall survival (OS) and nutritional status and inflammation factors were examined using Kaplan-Meier curves and logrank tests. Results: Serum albumin, cholesterol and Creactive protein concentration, neutrophil count and platelet count were shown to be correlated with OS. We constructed a new risk model (nutrition inflammation status, NIS) using these factors, and compared it with other nutrition and inflammation models. Conclusion: NIS was useful as a new model for predicting $O S$ in patients undergoing curative resection for colorectal cancer, compared with known models.
\end{abstract}

In recent years, as typified by the term 'precision medicine', identification of risk factors for recurrence according to individual conditions and formulation of a classification for recurrence risk which exceeds the TNM classification have been sought. Recent studies have reported that the preoperative nutritional status and internal inflammatory findings as patient factors are correlated with postoperative recurrence risk, and that diets that cause colorectal

This article is freely accessible online.

Correspondence to: Dr. Tamuro Hayama, Department of Surgery, Teikyo University School of Medicine, 2-11-1 Kaga, Itabashi-ku, Tokyo 173-8605, Japan. Tel: +81 339641211, Fax: +81353756097, e-mail: tamuro@med.teikyo-u.ac.jp

Key Words: Colon cancer, NLR, CAR, PNI, GPS, NIS. inflammation increase the risk of colorectal cancer (CRC). This suggests a relationship between inflammation and CRC (1-3). Clinicians need a simple prognostic tool for CRC. Simple prognostic tools have been developed using inflammatory markers and nutritional indices. Control of Nutritional Status (CONUT), which consists of serum albumin, total lymphocyte count, and serum total cholesterol level, Glasgow prognostic score (GPS) and prognostic nutritional index (PNI), is a widely established prognostic predictor for patients with cancer (4-6). However, its accuracy is still insufficient and there is no classification ability that exceeds the TNM classification. In this study, we aimed to develop a tool using nutritional indicators and inflammatory markers that indicate excellent overall survival (OS).

\section{Patients and Methods}

Patient selection. We enrolled 286 consecutive patients with stage I-III CRC, diagnosed based on the eighth edition of the United States Joint Commission on Cancer staging system (7), who underwent radical resection at Teikyo University Hospital in Japan between 2013 and 2017. This study was approved by Teikyo University Committee (registration number: 19-153) and written informed consent was obtained from all participants. The reporting of our research is in accordance with the STROBE guidelines (8).

Study metrics. The following parameters were determined for each patient. The variables evaluated included patient age, gender, body mass index (BMI), histological grade, tumor, lymph node, metastasis (TNM) stage, individual medical history, preoperative test data, and follow-up. Patients were excluded if they had multiple primary malignancies, or had familial adenomatous polyposis or Lynch syndrome. The histopathological, clinical, and laboratory data were obtained from the patients, and blood sampling as conducted within 1-2 weeks prior to the surgery.

GPS score: The GPS score was determined based on serum albumin and C-reactive protein (CRP) levels as follows: Score 2: hypoalbuminemia $(<3.5 \mathrm{~g} / \mathrm{dl})$ and the elevated $\mathrm{C}$-reactive protein $(\mathrm{CRP})(>1.0 \mathrm{mg} / \mathrm{dl})$; score 1 : hypoalbuminemia $(<3.5 \mathrm{~g} / \mathrm{dl})$ or CRP $>1.0 \mathrm{mg} / \mathrm{dl}$; score 0 : albumin concentration $\geq 3.5 \mathrm{~g} / \mathrm{dl}$ and $\mathrm{CRP} \leq 1.0 \mathrm{mg} / \mathrm{dl}(9)$. 
PNI: The PNI was calculated using serum albumin and the peripheral lymphocyte count as a simple and useful score for predicting the prognosis for patients with various cancer types (10), where $\mathrm{PNI}=$ serum albumin level $(\mathrm{g} / \mathrm{dl})+5 \times$ total lymphocyte count (11). Onodera et al. reported that this index provided an accurate, quantitative estimate of operative risk (12). In general, resection and anastomosis of the gastrointestinal tract can be safely practiced when the index is $>45$. The same procedure may be dangerous when the index is between 45 and 40 . When the PNI is $<40$, this form of surgery may be contraindicated (12).

Indices of general condition. The neutrophil:lymphocyte ratio (NLR) and C-reactive protein:albumin ratio (CAR) were determined as indices of patients'general condition.

The NLR, CAR, GPS score and PNI score were obtained from data derived from analysis blood samples taken within 1-2 weeks preoperatively.

Follow-up. Surgical resection was defined as curative when there was no evidence of tumor recurrence and the distant metastases were histologically and macroscopically complete. Patients were followed up every 3 months for the first 3 years, and every 6 months for the next 2 years. At each follow-up, all patients underwent physical examination and measurements of serum carcinoembryonic antigen (CEA) and carbohydrate antigen 19-9 (CA19-9). They also underwent colonoscopy 1-2 years after surgery (for rectal cancer, every year after surgery). Thoracoabdominal computed tomography scans were usually taken every 6 months. Recurrence was defined as the appearance of a radiological, clinical, or pathological diagnosis of cancer cells that were local or distant from their original location.

Determination of cut-off values for the nutrition inflammation status (NIS)-based risk model. The continuous parameters were assessed based on the receiver operating characteristic (ROC) curve, and the cut-off value for each parameter was set using the Youden-index. The comparison was made by dividing patients into two groups, a highvalue group and a low-value group. For each parameter, a ROC curve was created by plotting the sensitivity and specificity of each result under investigation. The score closest to the point with both maximum sensitivity and specificity was selected as the cut-off value.

The interpretation of an index of probability of concordance (Cindex) between predicted probability and actual outcome was used so as to evaluate the predictive ability and discrimination of the model. The value of the C-index should fall between 0.5 and 1.0, with 0.5 indicating random chance and 1.0 indicating a perfect discriminative ability ( $\mathrm{R}$ version 4.0.3 software).

We also evaluated our data using the Akaike information criterion (AIC), which is a popular method for comparing the adequacy of multiple, possibly non-nested models (13). It is a statistic that evaluates the predictability of a statistical model using the difference between the observed value and the theoretical value. The smaller the AIC value, the better is the fit of the model (14).

Statistical analysis. OS was calculated from the date the patient underwent surgery to that of death, using the Kaplan-Meier method. A Cox regression analysis was performed to identify factors significantly associated with OS. $p$-Values of 0.05 or less were considered significant. All statistical analyses were performed using JMP 15 software (SAS, Cary, NC, USA).
Table I. Clinicopathological features of patients with stage I-III colorectal cancer who underwent curative tumor resection.

\begin{tabular}{|c|c|c|c|}
\hline $\begin{array}{l}\text { Clinicopathological } \\
\text { feature }\end{array}$ & Subgroup & $\mathrm{N}=286(\%)$ & $\begin{array}{l}\text { Cut-off } \\
\text { value }\end{array}$ \\
\hline \multirow[t]{2}{*}{ Gender, n (\%) } & Male & $166(58.0)$ & - \\
\hline & Female & $120(42.0)$ & \\
\hline Age, years & Median (range) & $67.5(28-93)$ & - \\
\hline \multirow[t]{2}{*}{ BMI, $\mathrm{kg} / \mathrm{m}^{2}$} & Low & $68(76.2)$ & 25.2 \\
\hline & High & $218(23.8)$ & \\
\hline \multirow[t]{2}{*}{ T Stage, n (\%) } & $\mathrm{T} 1,2$ & $97(33.9)$ & - \\
\hline & $\mathrm{T} 3,4$ & $189(66.1)$ & \\
\hline \multirow[t]{2}{*}{ N Stage, n (\%) } & $\mathrm{N}+$ & $106(37.0)$ & - \\
\hline & - & $180(62.9)$ & \\
\hline \multirow[t]{2}{*}{$\mathrm{CEA}, \mathrm{ng} / \mathrm{ml}$} & Low & $137(47.9)$ & 3.5 \\
\hline & High & $149(52.1)$ & \\
\hline \multirow[t]{2}{*}{ CA19-9, U/ml } & Low & $252(88.1)$ & 50.2 \\
\hline & High & $34(11.9)$ & \\
\hline \multirow[t]{2}{*}{ Albumin, g/dl } & Low & $125(43.7)$ & 3.9 \\
\hline & High & $161(56.2)$ & \\
\hline \multirow[t]{2}{*}{ Lymphocytes, $\mathrm{n} / \mathrm{mm}^{3}$} & Low & $190(66.4)$ & 1,606 \\
\hline & High & $96(33.6)$ & \\
\hline \multirow[t]{2}{*}{ Cholesterol, mg/dl } & Low & $14(4.9)$ & 119 \\
\hline & High & $272(95.1)$ & \\
\hline \multirow[t]{2}{*}{ WBC, $\mathrm{n} / \mu \mathrm{l}$} & Low & $146(51.0)$ & 6,000 \\
\hline & High & $140(49.0)$ & \\
\hline \multirow[t]{2}{*}{ Neutrophils, $\mathrm{n} / \mathrm{mm}^{3}$} & Low & $184(64.3)$ & 4,307 \\
\hline & High & $102(35.6)$ & \\
\hline \multirow[t]{2}{*}{ Platelets, $\mathrm{n} / \mathrm{mm}^{3}$} & Low & $243(85.0)$ & 19.5 \\
\hline & High & $43(15.0)$ & \\
\hline \multirow[t]{2}{*}{$\mathrm{CRP}, \mathrm{mg} / \mathrm{dl}$} & Low & $168(58.7)$ & 0.12 \\
\hline & High & $118(41.3)$ & \\
\hline \multirow[t]{2}{*}{ NLR, n (\%) } & Low & $86(30.0)$ & 2.24 \\
\hline & High & $200(70.0)$ & \\
\hline \multirow[t]{2}{*}{ CAR, n (\%) } & Low & $132(46.6)$ & 0.025 \\
\hline & High & $154(53.8)$ & \\
\hline \multirow[t]{2}{*}{ PNI, n (\%) } & Low & $154(53.8)$ & 46.23 \\
\hline & High & $132(46.1)$ & \\
\hline \multirow[t]{3}{*}{ GPS, n (\%) } & 0 & $196(68.5)$ & - \\
\hline & 1 & $59(20.6)$ & \\
\hline & 2 & $31(10.8)$ & \\
\hline
\end{tabular}

BMI: Body mass index; CA19-9: carbohydrate antigen 19-9; CAR: Creactive protein (CRP):albumin ratio; CEA: carcinoembryonic antigen; GPS: Glasgow prognostic score; NLR: neutrophil-lymphocyte ratio; PNI: prognostic nutritional index; WBC: white blood cells.

\section{Results}

Patient characteristics. The study included a total of 286 patients. The median age was 67.5 (range=28-93) years; 166 (58.0\%) patients were male. Most patients had T3 or T4 tumor $(n=189,66.1 \%)$. There were $106(37.0 \%)$ cases with lymph node metastasis and GPS was 0 for most patients $(196,68.5 \%)$ (Table I).

Determination of cut-off values. ROC curve analysis was used to determine cut-off values for WBC, neutrophils, 

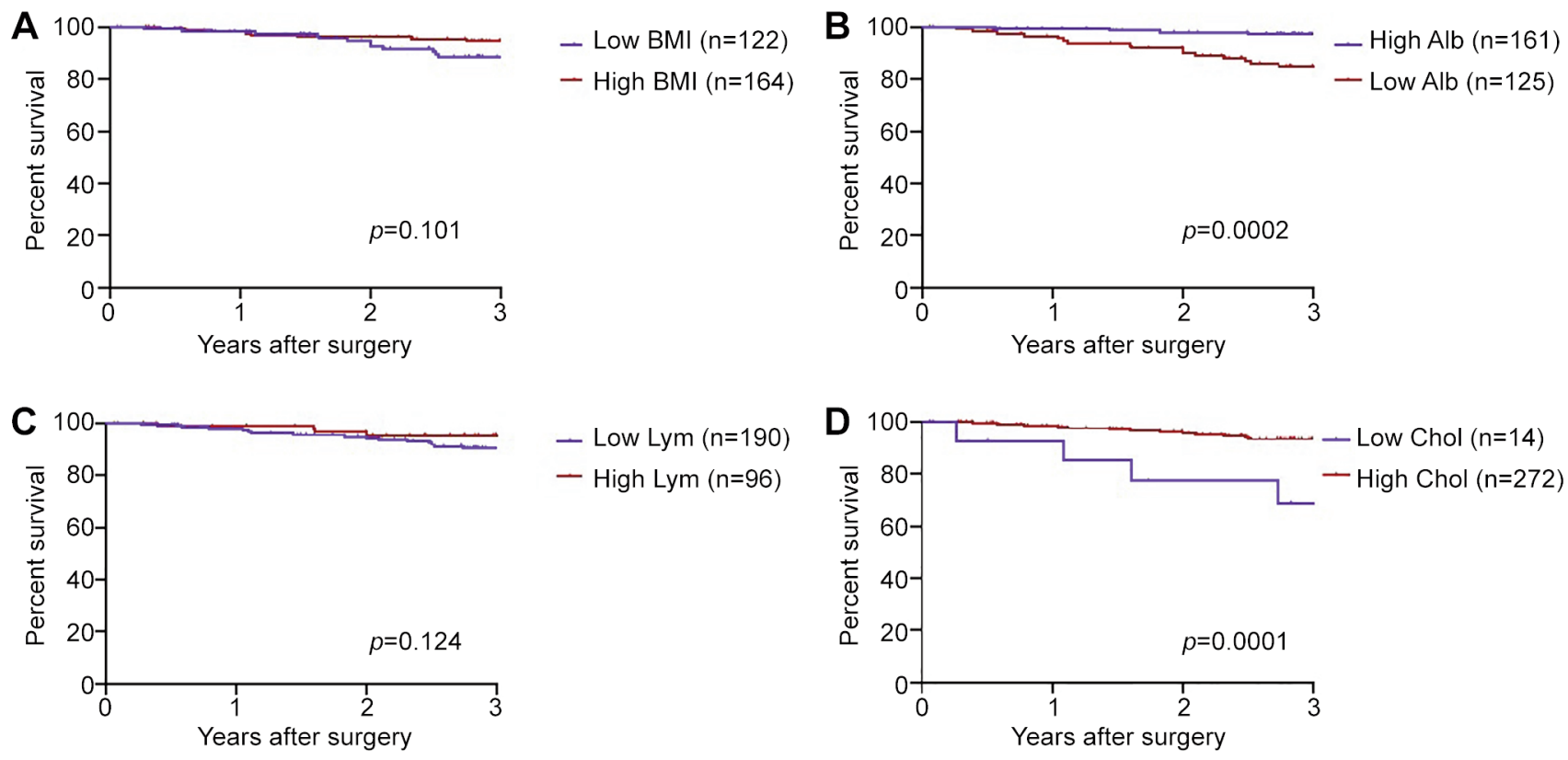

Figure 1. Kaplan-Meier curves of 3-year overall survival based on the indices body mass index (BMI) (A), serum albumin (Alb) (B), lymphocyte count (Lym) (C), and serum cholesterol (Chol) (D).

platelets, albumin, lymphocytes, cholesterol, NLR, and CAR for predicting 3-year-OS (Table I).

Relationship between nutritional findings and OS. BMI, lymphocyte count, serum albumin and cholesterol were used as nutritional indicators. The Kaplan-Meier curves of the OS according to each of these parameters are shown in Figure 1. BMI and lymphocyte count were not significant factors. On the other hand, the group with a low albumin level had a significantly worse OS than the high-albumin group $(p<0.0002)$. Similarly, significantly worse OS was found for the low-cholesterol group than in the high-cholesterol group $(p<0.0001)$ (Figure 1).

Relationship between Inflammatory findings and OS. White blood cell count (WBC), neutrophil count, platelet count, and CRP were used as inflammatory findings. The Kaplan-Meier curves of the OS according to each of these parameters are shown in Figure 2. The WBC were not a significant factor in the relationship for overall survival $(p=0.234)$. On the other hand, for the groups with high neutrophil count, platelet count, and CRP level, the OS was significantly worse than that of the low-value groups. $(p=0.017, p=0.006$ and $p=0.0003$, respectively) (Figure 2).

Relationship between NLR, CAR, PNI, tumor markers and $O S$. As known models, NLR and CAR were used as inflammation indicators, and PNI and GPS were used as nutritional indicators. NLR was not a significant factor in OS $(p=0.051)$. CAR, PNI, and GPS were indicators significantly associated with OS $(p=0.0001, p<0.0001$ and $p=0.006)$ (Figure 3). CEA and CA19-9 were used as tumor markers. CA19-9 was not a significant factor in OS $(p=0.427)$; on the other hand, the OS was significantly worse in the high-CEA group than in the low-CEA group $(p=0.002)$.

Stratification using patient factors. Univariate analysis was performed using the Cox proportional hazard model for the factors of BMI, albumin, lymphocyte count, cholesterol, WBC, neutrophil count, platelet count, serum albumin and cholesterol, and CRP in the OS (Table II). We decided to create a new risk model, namely the nutrition inflammation status (NIS), using the five factors that were significantly associated with OS, namely albumin, cholesterol, neutrophil count, platelet count, and CRP. The risk model was calculated by adding up the above-mentioned number of risk factors, in which the NIS is the sum of the number of risk factors. Kaplan-Meier survival curve and set the cut-off value using the Youden index (Figure 4A). As a result, an NIS of 3 or more assigned the patient to the high-value group and an NIS of 2 or less to the low-value group.

Kaplan-Meier OS curve according to NIS. Survival analyses were performed according to NIS using the cut-off value to divide patients into two groups. A statistically significant difference in 3-year OS between the two groups was revealed by Kaplan-Meier curves $(p<0.0001)$, indicating the potential prognostic value of NIS. The 3-year OS rates were 

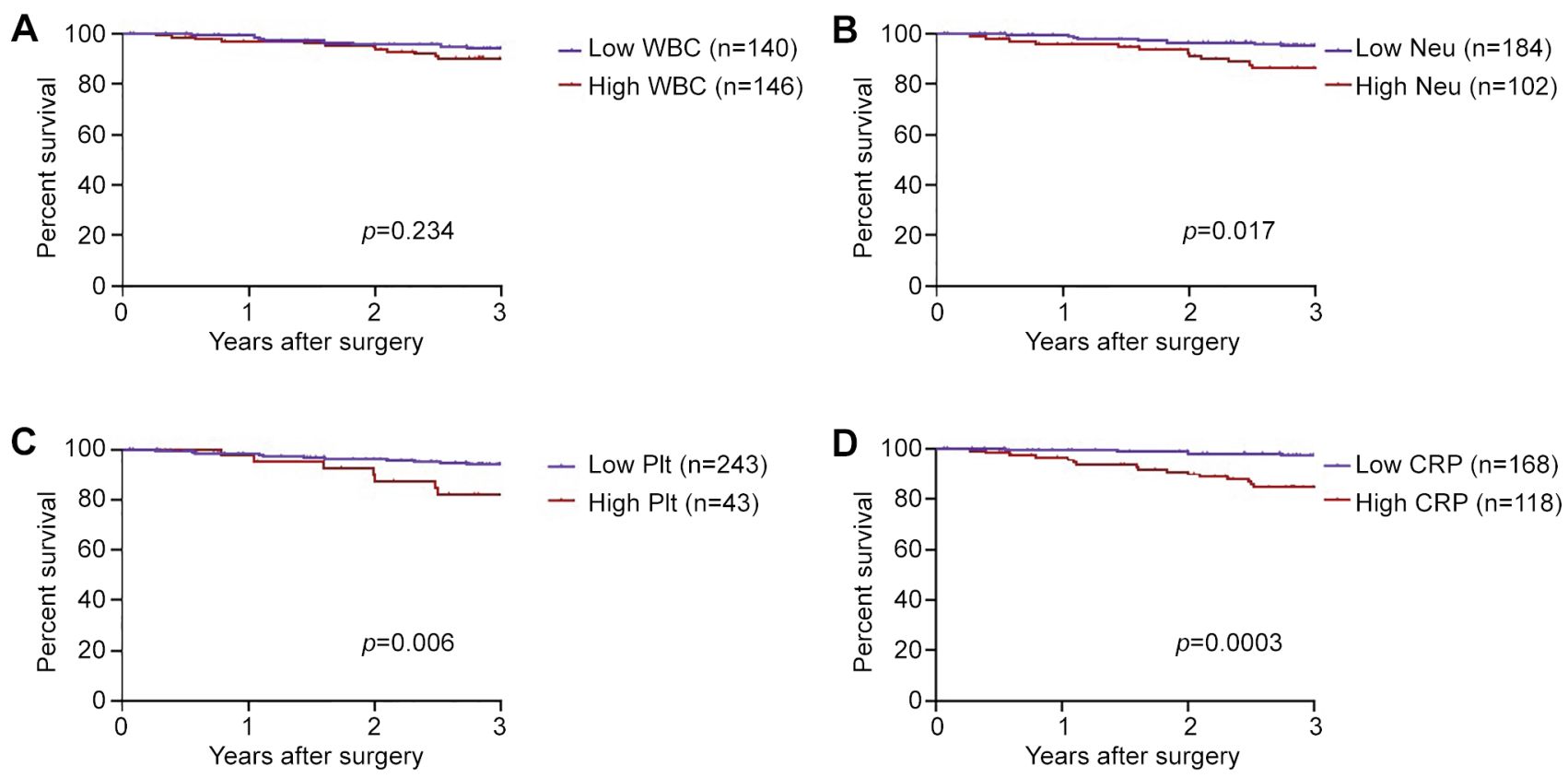

Figure 2. Kaplan-Meier curves of 3-year overall survival based on the inflammatory markers. (A) white blood cells (WBC), (B) neutrophil count $(\mathrm{Neu}),(\mathrm{C})$ platelet count $(\mathrm{Plt})$, and $(\mathrm{D}) \mathrm{C}$-reactive protein $(\mathrm{CRP})$.
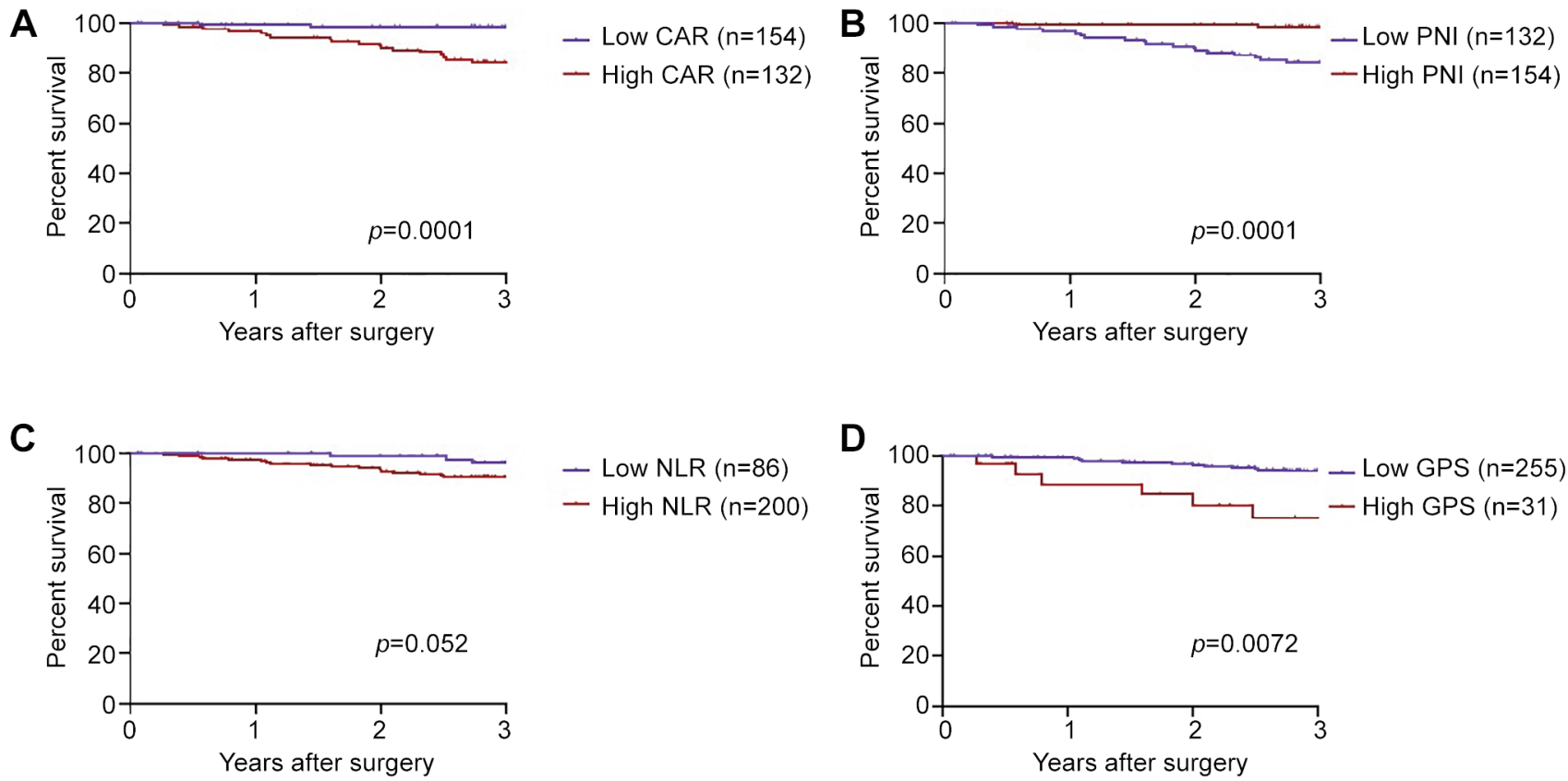

Figure 3. Kaplan-Meier curves of 3-year overall survival based on the known nutritional and inflammatory markers C-reactive protein:albumin ratio $(C A R)(A)$, prognostic nutritional index $(P N I)(B)$, neutrophil:lymphocyte ratio $(N L R)(C)$, and Glasgow prognostic score $(G P S)(D)$.

$97.1 \%$ for the low-NIS group and $77.3 \%$ for the high-NIS group, showed significantly poorer prognosis for the highNIS group (Figure 4B).
Evaluation of NIS using C-index and AIC compared with $T$ factor and $N$ factor, CAR, PNI, GPS and CEA. Univariate and multivariate analyses were performed using the Cox 
Table II. Univariate analysis of nutritional and inflammatory findings using Cox proportional hazard model for 3-year overall survival.

\begin{tabular}{lllcl}
\hline Factor & Comparison & HR & $95 \%$ CI & $p$-Value \\
\hline BMI & Low $v s$. high & 2.0 & $0.70-5.9$ & 0.161 \\
Albumin & Low $v s$. high & 4.2 & $1.8-9.9$ & $\mathbf{0 . 0 0 0 4}$ \\
Lymphocyte & Low $v s$. high & 2.23 & $0.8-5.9$ & 0.105 \\
$\quad$ count & & & & \\
Cholesterol & Low $v$ s. high & 5.8 & $2.2-15.6$ & $\mathbf{0 . 0 0 3}$ \\
WBC & High $v s$. low & 1.59 & $0.7-3.4$ & 0.234 \\
Neutrophil & High $v$ s. low & 2.45 & $1.1-5.2$ & $\mathbf{0 . 0 2}$ \\
count & & & & \\
Platelet count & High $v s$. low & 2.9 & $1.3-6.5$ & $\mathbf{0 . 0 1 5}$ \\
CRP & High $v s$. low & 4.33 & $1.8-10.3$ & $\mathbf{0 . 0 0 0 3}$ \\
\hline
\end{tabular}

BMI: Body mass index; CRP: C-reactive protein; WBC: white blood cells. Statistically significant $p$-values are shown in bold.

proportional hazard model to determine the association of $\mathrm{T}$ factor and $\mathrm{N}$ factor, CAR, PNI, GPS, CEA, and NIS with OS. As a result of multivariate analysis, only three significant factors were extracted as being associated with OS: TNM, PNI and NIS (HR=2.9, 95\% confidence interval $(\mathrm{CI})=1.3-6.5 ; \mathrm{HR}=4.8,95 \% \mathrm{CI}=1.50-15.5$; and $\mathrm{HR}=4.4,95 \%$ $\mathrm{CI}=1.5-12.9$, respectively). As shown in Table III, C-indices for TNM, PNI and NIS for predicting OS were $0.636,0.726$ and 0.747 , respectively. The AIC values for OS were 277.85 for TNM, 265.32 for PNI, 257.34 for NIS. According to this comparison, NIS had the best goodness-of-fit, followed by TNM and PNI. NIS was the best model to reflect the prognosis after surgery for CRC (Table III).

\section{Discussion}

Currently, there are various indicators that clinically indicate the nutritional status of patients and the findings of inflammation in the body. These include the serum protein level, serum albumin level, serum prealbumin level, serum transferrin level, and lymphocyte count as markers indicating nutritional status, and the GPS and PNI that combine these markers. It has been reported that BMI (14), NLR (15), CAR (16), PNI (17), and GPS (18) are factors related to the prognosis of patients undergoing curative resection for CRC. We conducted a study with the aim of constructing a new model for selection of a group at high risk of recurrence, by combining tumor factors and preoperative nutritional status as patient factors, with findings of an inflammatory state.

The items used in this new risk model were five factors that were significantly associated with OS: albumin, cholesterol, neutrophil count, platelet count and CRP. Each of the five factors has been reported to be associated with OS. Albumin is produced by hepatocytes, and reflects the nutritional status and amount of skeletal muscle, and is decreased by inflammatory mediators $(15,16)$. Hypoalbuminemia often may reflect the presence of progressive disease and poor performance status caused by tumor cachexia. The role of pretreatment serum albumin as a prognostic factor was demonstrated by several studies (17-19). Cholesterol is widely distributed not only in the blood but also throughout the body such as the brain, internal organs, muscles, and bile (20). The serum total cholesterol level is known to be an indicator of a patient's reserve calories (21). Other studies have reported that a low serum cholesterol level confers a poor prognosis for patients with various cancer types (22-24). The relationship between low total blood cholesterol and cancer mortality has been reported in many previous studies. It is thought that this is mainly because the total cholesterol level decreases with the development of cancer, especially with the progression of colorectal cancer and advanced cancer (25). Therefore, hypocholesterolemia is not considered to be the cause of cancer, but it is thought to be caused by cancer (24).

In this study, a factor for poor prognosis was a high number of neutrophils. The mechanism underlying the association between a high number of neutrophils and worse outcomes has not yet been completely clarified. However, it might be attributed to the association of neutrophils with inflammation. Neutrophils can inhibit the immune system, thereby eliminating the cytolytic activity of immune cells $(26,27)$. Both tumor and host cells, including neutrophils, can simultaneously produce chemokines and cytokines, thus contributing to tumor progression (28).

Rao et al. reported that an elevated platelet count is a negative predictor of survival in both patients with primary CRC and those with resectable colorectal liver metastases (29). Studies have shown that an increased platelet count promotes the growth, infiltration, and metastasis of cancer (30-32). Platelets can secrete a variety of growth factors and angiogenesis-regulatory proteins (interleukin-6, vascular endothelial growth factor, platelet-derived growth factor, platelet factor 4) to promote tumor formation and metastasis (33).

Allin $e t a l$. reported that patients with invasive breast cancer and diagnostic CRP levels $>3 \mathrm{mg} / \mathrm{l}$ had a 1.7 -fold increased risk of death from breast cancer compared to patients with levels $<1 \mathrm{mg} / \mathrm{l}$ (30). Biological mechanisms associated with an elevated CRP level and poor cancer prognosis may explain inflammation in the tumor microenvironment. Inflammation of the tumor microenvironment induces DNA damage, promotes angiogenesis, and promotes tumor spread and distant metastasis. The result is a tumorigenesis-promoting environment (34-36).

Currently, TNM staging is most frequently used to predict survival outcomes and treatment choices. However, because TNM staging is done postoperatively, survival cannot be predicted preoperatively. Moreover, no further treatment strategy can be determined. In addition, the TNM stage can 
A

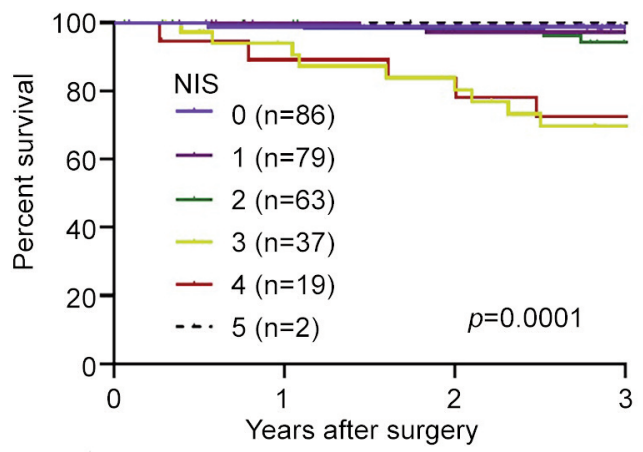

B

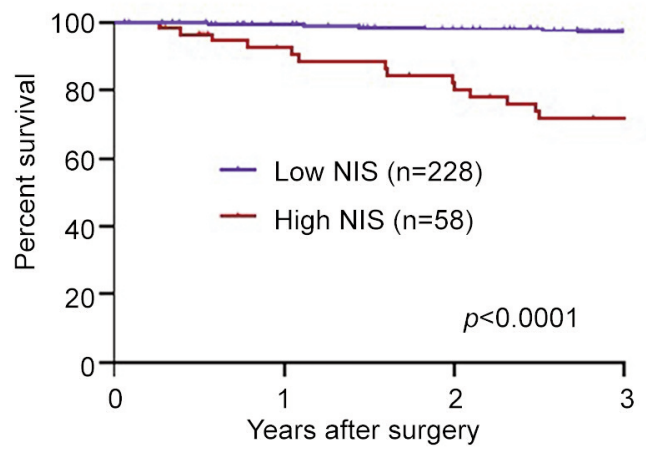

Figure 4. Kaplan-Meier curves of 3-year overall survival based on the nutritional and inflammation status (NIS) (A) and using a cut-off dividing patients into groups with low ( $\leq 2)$ and high ( $\geq 3)$ NIS (B).

Table III. Results of univariate and multivariate analyses for 3-year overall survival using Cox proportional hazard model, C-index and Akaike's information criterion (AIC).

\begin{tabular}{|c|c|c|c|c|c|c|c|c|c|}
\hline Factor & Comparison & HR & $95 \% \mathrm{CI}$ & $p$-Value & HR & $95 \% \mathrm{CI}$ & $p$-Value & C-Index & AIC \\
\hline TNM & Low vs. high & 13.8 & $1.9-102$ & 0.01 & & & & & \\
\hline CAR & Low vs. high & 5.5 & $2.1-14.4$ & 0.001 & & & & & \\
\hline PNI & High vs. low & 7.5 & $2.6-21.6$ & 0.0002 & 4.8 & $1.5-15.5$ & 0.009 & 0.726 & 265.32 \\
\hline GPS & Low vs. high & 4.2 & $1.6-10.9$ & 0.011 & & & & & \\
\hline NIS & Low vs. high & 8.3 & $3.8-18.1$ & $<0.0001$ & 4.4 & $1.5-12.9$ & 0.008 & 0.747 & 257.34 \\
\hline
\end{tabular}

CAR: C-Reactive protein:albumin ratio; GPS: Glasgow prognostic score; NIS: nutritional and Inflammation status; PNI: prognostic nutritional index; TNM: tumor node metastasis. Statistically significant $p$-values are shown in bold.

only reflect the biological behavior of the tumor. The prognosis of cancer is associated with nutritional status and the inflammatory response of the host $(1,37)$. The NIS is based on peripheral neutrophil and platelet counts, and the levels of CRP, albumin and cholesterol, and reflects the state of the tumor microenvironment and the preoperative host inflammatory response and nutritional status. Our findings show that the preoperative NIS has a stronger prognostic discriminative capability compared to the NLR, CAR, GPS, and PNI. Therefore, the use of a combination of parameters that reflects both the nutritional status and the systemic inflammatory status may be important for accurately predicting survival outcomes in patients with CRC.

This study has its limitations. Firstly, it was retrospective in design and included patients from a single institution. Secondly, this study had a small sample size. Thirdly, there is no consensus regarding the nutrition and inflammatory marker cut-off values. We selected cut-off values by performing a ROC analysis. Our current findings require further review and validation in more patients. Fourthly, the results of this study do not apply to patients with stage IV disease, as it only included those with stage I-III CRC who underwent curative surgery.

\section{Conclusion}

NIS using nutritional indicators and inflammatory findings was found to be useful as a new model for predicting OS in patients undergoing curative resection for CRC, compared with known models.

\section{Conflicts of Interest}

The Authors declare that they have no conflicts of interest.

\section{Authors' Contributions}

TH, TO, MT, YF, RS, KN, KM, SF, TF and YH were involved in study design and data interpretation. TH, TO, MT, YF, RS, KN, $\mathrm{KM}, \mathrm{SF}, \mathrm{TF}$ and $\mathrm{YH}$ were involved in the data analysis. All Authors critically revised the report, commented on drafts of the article, and approved the final report.

\section{Acknowledgements}

The Authors are grateful for $20^{\text {th }}$ Fujii Tomoko Academic Encouragement Award and ACRO Research Grants of Teikyo University in the early stages of this work. 


\section{References}

1 Hayama T, Ozawa T, Okada Y, Tsukamoto M, Fukushima Y, Shimada R, Nozawa K, Matsuda K, Fujii S and Hashiguchi Y: The pretreatment Controlling Nutritional Status (CONUT) score is an independent prognostic factor in patients undergoing resection for colorectal cancer. Sci Rep 10(1): 13239, 2020 PMID: 32764671. DOI: 10.1038/s41598-020-70252-2

2 Chen JH, Zhai ET, Yuan YJ, Wu KM, Xu JB, Peng JJ, Chen CQ, He YL and Cai SR: Systemic immune-inflammation index for predicting prognosis of colorectal cancer. World J Gastroenterol 23(34): 6261-6272, 2017. PMID: 28974892. DOI: 10.3748/ wjg.v23.i34.6261

3 Zhang J, Zhang HY, Li J, Shao XY and Zhang CX: The elevated NLR, PLR and PLT may predict the prognosis of patients with colorectal cancer: a systematic review and meta-analysis. Oncotarget 8(40): 68837-68846, 2017. PMID: 28978160. DOI 10.18632 /oncotarget.18575

4 Minami S, Ogata Y, Ihara S, Yamamoto S and Komuta K: Pretreatment Glasgow prognostic score and prognostic nutritional index predict overall survival of patients with advanced small cell lung cancer. Lung Cancer (Auckl) 8: 249257, 2017. PMID: 29263709. DOI: 10.2147/LCTT.S142880

5 Miyake M, Morizawa Y, Hori S, Marugami N, Iida K, Ohnishi K, Gotoh D, Tatsumi Y, Nakai Y, Inoue T, Anai S, Torimoto K, Aoki K, Tanaka N, Shimada K, Konishi N and Fujimoto K: Integrative assessment of pretreatment inflammation-, nutrition, and muscle-based prognostic markers in patients with muscleinvasive bladder cancer undergoing radical cystectomy. Oncology 93(4): 259-269, 2017. PMID: 28647740. DOI: $10.1159 / 000477405$

6 Watanabe J, Otani S, Sakamoto T, Arai Y, Hanaki T, Amisaki M, Tokuyasu N, Honjo S and Ikeguchi M: Prognostic indicators based on inflammatory and nutritional factors after pancreaticoduodenectomy for pancreatic cancer. Surg Today 46(11): 1258-1267, 2016. PMID: 26869184. DOI: 10.1007/ s00595-016-1308-6

7 Weiser MR: AJCC $8^{\text {th }}$ edition: Colorectal cancer. Ann Surg Oncol 25(6): 1454-1455, 2018. PMID: 29616422. DOI: 10.1245/ s10434-018-6462-1

8 von Elm E, Altman DG, Egger M, Pocock SJ, Gøtzsche PC, Vandenbroucke JP and STROBE Initiative: The Strengthening the Reporting of Observational Studies in Epidemiology (STROBE) Statement: guidelines for reporting observational studies. Int J Surg 12(12): 1495-1499, 2014. PMID: 25046131 DOI: $10.1016 / j . i j s u .2014 .07 .013$

9 McMillan DC, Elahi MM, Sattar N, Angerson WJ, Johnstone J and McArdle CS: Measurement of the systemic inflammatory response predicts cancer-specific and non-cancer survival in patients with cancer. Nutr Cancer 41(1-2): 64-69, 2001. PMID: 12094630. DOI: 10.1080/01635581.2001.9680613

10 Tominaga T, Nonaka T, Hisanaga M, Fukuda A, Tanoue Y, Yoshimoto T, Hidaka S, Sawai T and Nagayasu T: Prognostic value of the preoperative prognostic nutritional index in oldestold patients with colorectal cancer. Surg Today 50(5): 449-459, 2020. PMID: 31720800. DOI: 10.1007/s00595-019-01910-w

11 Nozoe T, Kohno M, Iguchi T, Mori E, Maeda T, Matsukuma A and Ezaki T: The prognostic nutritional index can be a prognostic indicator in colorectal carcinoma. Surg Today 42(6): 532-535, 2012. PMID: 22139087. DOI: 10.1007/s00595-011-0061-0
12 Onodera T, Goseki N and Kosaki G: [Prognostic nutritional index in gastrointestinal surgery of malnourished cancer patients]. Nihon Geka Gakkai Zasshi 85(9): 1001-1005, 1984. PMID: 6438478.

13 Vrieze SI: Model selection and psychological theory: a discussion of the differences between the Akaike information criterion (AIC) and the Bayesian information criterion (BIC). Psychol Methods 17(2): 228-243, 2012. PMID: 22309957. DOI: 10.1037/a0027127

14 Akaike H: A new look at the statistical model identification. IEEE Transactions on Automatic Control 19(6): 716-723, 2017. DOI: 10.1109/TAC.1974.1100705

15 Visser M, Kritchevsky SB, Newman AB, Goodpaster BH, Tylavsky FA, Nevitt MC and Harris TB: Lower serum albumin concentration and change in muscle mass: the Health, Aging and Body Composition Study. Am J Clin Nutr 82(3): 531-537, 2005. PMID: 16155264. DOI: 10.1093/ajcn.82.3.531

16 Fuhrman MP: The albumin-nutrition connection: separating myth from fact. Nutrition 18(2): 199-200, 2002. PMID: 11844655. DOI: 10.1016/s0899-9007(01)00729-8

17 Ayhan A, Günakan E, Alyazıcı İ, Haberal N, Altundağ Ö and Dursun P: The preoperative albumin level is an independent prognostic factor for optimally debulked epithelial ovarian cancer. Arch Gynecol Obstet 296(5): 989-995, 2017. PMID: 28875365. DOI: 10.1007/s00404-017-4511-9

18 Fan Y, Xiang S, Dai Z, Zou C, Wang X and Gao Z: Prognostic significance of $\mathrm{C}$-reactive protein to albumin ratio in colorectal cancer patients: a meta-analysis. Int J Colorectal Dis 34(6): 1105-1111, 2019. PMID: 31016379. DOI: 10.1007/s00384-01903299-x

19 Yamamoto M, Saito H, Uejima C, Tanio A, Tada Y, Matsunaga T, Sakamoto T, Honjo S, Ashida K and Fujiwara Y: Combination of serum albumin and cholinesterase levels as prognostic indicator in patients with colorectal cancer. Anticancer Res 39(2): 10851090, 2019. PMID: 30711999. DOI: 10.21873/anticanres.13217

20 Kuzu OF, Noory MA and Robertson GP: The role of cholesterol in cancer. Cancer Res 76(8): 2063-2070, 2016. PMID: 27197250. DOI: 10.1158/0008-5472.CAN-15-2613

21 Gadgil MD, Anderson CA, Kandula NR and Kanaya AM: Dietary patterns are associated with metabolic risk factors in South Asians living in the United States. J Nutr 145(6): 12111217, 2015. PMID: 25904730. DOI: 10.3945/jn.114.207753

22 Ko K, Park YH, Lee JW, Ku JH, Kwak C and Kim HH: Influence of nutritional deficiency on prognosis of renal cell carcinoma (RCC). BJU Int 112(6): 775-780, 2013. PMID: 24028765. DOI: 10.1111/bju.12275

23 Cubiella J, Castells A, Fondevila C, Sans M, Sabater L, Navarro $\mathrm{S}$ and Fernández-Cruz L: Prognostic factors in nonresectable pancreatic adenocarcinoma: a rationale to design therapeutic trials. Am J Gastroenterol 94(5): 1271-1278, 1999. PMID: 10235206. DOI: 10.1111/j.1572-0241.1999.01018.x

24 Zhou P, Li B, Liu B, Chen T and Xiao J: Prognostic role of serum total cholesterol and high-density lipoprotein cholesterol in cancer survivors: A systematic review and meta-analysis. Clin Chim Acta 477: 94-104, 2018. PMID: 29223765. DOI: 10.1016/ j.cca.2017.11.039

25 de Ulíbarri Pérez JI, Fernández G, Rodríguez Salvanés F and Díaz López AM: Nutritional screening; control of clinical undernutrition with analytical parameters. Nutr Hosp 29(4): 797811, 2014. PMID: 24679020. DOI: 10.3305/nh.2014.29.4.7275 
26 el-Hag A and Clark RA: Immunosuppression by activated human neutrophils. Dependence on the myeloperoxidase system. J Immunol 139(7): 2406-2413, 1987. PMID: 2821114.

27 Petrie HT, Klassen LW and Kay HD: Inhibition of human cytotoxic $\mathrm{T}$ lymphocyte activity in vitro by autologous peripheral blood granulocytes. J Immunol 134(1): 230-234, 1985. PMID: 3871101.

28 Dell' Aquila E, Cremolini C, Zeppola T, Lonardi S, Bergamo F, Masi G, Stellato M, Marmorino F, Schirripa M, Urbano F, Ronzoni M, Tomasello G, Zaniboni A, Racca P, Buonadonna A, Allegrini G, Fea E, Di Donato S, Chiara S, Tonini G, Tomcikova D, Boni L, Falcone A and Santini D: Prognostic and predictive role of neutrophil/lymphocytes ratio in metastatic colorectal cancer: a retrospective analysis of the TRIBE study by GONO. Ann Oncol 29(4): 924-930, 2018. PMID: 29324972. DOI: 10.1093/annonc/mdy004

29 Rao XD, Zhang H, Xu ZS, Cheng H, Shen W and Wang XP: Poor prognostic role of the pretreatment platelet counts in colorectal cancer: A meta-analysis. Medicine (Baltimore) 97(23): e10831, 2018. PMID: 29879017. DOI: 10.1097/MD.0000000000010831

30 Riedl J, Pabinger I and Ay C: Platelets in cancer and thrombosis. Hamostaseologie 34(1): 54-62, 2014. PMID: 24305775. DOI: 10.5482/HAMO-13-10-0054

31 Radziwon-Balicka A, Santos-Martinez MJ, Corbalan JJ, O'Sullivan S, Treumann A, Gilmer JF, Radomski MW and Medina C: Mechanisms of platelet-stimulated colon cancer invasion: role of clusterin and thrombospondin 1 in regulation of the P38MAPK-MMP-9 pathway. Carcinogenesis 35(2): 324332, 2014. PMID: 24085798. DOI: 10.1093/carcin/bgt332

32 Haemmerle M, Stone RL, Menter DG, Afshar-Kharghan V and Sood AK: The platelet lifeline to cancer: challenges and opportunities. Cancer Cell 33(6): 965-983, 2018. PMID: 29657130. DOI: $10.1016 /$ j.ccell.2018.03.002
33 Peterson JE, Zurakowski D, Italiano JE Jr, Michel LV, Connors S, Oenick M, D'Amato RJ, Klement GL and Folkman J: VEGF, PF4 and PDGF are elevated in platelets of colorectal cancer patients. Angiogenesis 15(2): 265-273, 2012. PMID: 22402885. DOI: $10.1007 / \mathrm{s} 10456-012-9259-\mathrm{z}$

34 Coussens LM and Werb Z: Inflammation and cancer. Nature 420(6917): 860-867, 2002. PMID: 12490959. DOI: 10.1038/ nature 01322

35 Vakkila $\mathrm{J}$ and Lotze MT: Inflammation and necrosis promote tumour growth. Nat Rev Immunol 4(8): 641-648, 2004. PMID: 15286730. DOI: $10.1038 /$ nri1415

36 Mantovani A, Allavena P, Sica A and Balkwill F: Cancer-related inflammation. Nature 454(7203): 436-444, 2008. PMID: 18650914. DOI: 10.1038 /nature07205

37 Hayama T, Hashiguchi Y, Okada Y, Ono K, Nemoto K, Shimada $R$, Ozawa $T$, Toyoda $T$, Tsuchiya $T$, Iinuma $H$, Nozawa $K$ and Matsuda K: Significance of the $7^{\text {th }}$ postoperative day neutrophilto-lymphocyte ratio in colorectal cancer. Int J Colorectal Dis 35(1): 119-124, 2020. PMID: 31797097. DOI: 10.1007/s00384019-03463-3
Received July 28, 2021

Revised October 20, 2021

Accepted October 25, 2021 